\title{
Management of Pediatric Inguinal Hernias in the Era of Laparoscopy: Results of an International Survey
}

\author{
Augusto Zani ${ }^{1,2}$ Simon Eaton ${ }^{1}$ Michael Hoellwarth ${ }^{3}$ Prem Puri ${ }^{4}$ Juan Tovar ${ }^{5}$ Guenter Fasching ${ }^{6}$ \\ Pietro Bagolan ${ }^{7}$ Marija Lukac ${ }^{8}$ Rene Wijnen ${ }^{9}$ Joachim Kuebler ${ }^{10}$ Giovanni Cecchetto ${ }^{11}$ \\ Risto Rintala ${ }^{12}$ Agostino Pierro ${ }^{1,13}$
}

${ }^{1}$ Department of Pediatric Surgery, University College London Institute of Child Health, London, United Kingdom

2 Department of Pediatric Surgery, Sapienza University of Rome, Rome, Italy

${ }^{3}$ Department of Pediatric and Adolescents Surgery, Medical University of Graz, Graz, Austria

${ }^{4}$ Department of Pediatric Surgery, National Children's Research Centre, Dublin, Ireland

${ }^{5}$ Department of Pediatric Surgery, Hospital Universitario La Paz, Madrid, Spain

6 Department of Pediatric Surgery, Klagenfurt General Hospital, Klagenfurt, Austria

${ }^{7}$ Department of Medical and Surgical Neonatology, Bambino Gesù Children's Hospital, Rome, Italy

${ }^{8}$ Department of Pediatric Surgery, Faculty of Medicine, University Children's Hospital, Belgrade, Serbia

${ }^{9}$ Department of Pediatric Surgery, Erasmus MC-Sophia in Erasmus MC Sophia Childrens' Hospital, Rotterdam, The Netherlands

${ }^{10}$ Department of Pediatric Surgery, Hannover Medical School, Hannover, Germany

${ }^{11}$ Department of Pediatric Surgery, University Hospital of Padua, Padova, Italy

${ }^{12}$ Department of Pediatric Surgery, Hospital for Children and Adolescents, Helsinki, Finland

13 Division of General and Thoracic Surgery, The Hospital for Sick Children, Toronto, Canada

Eur J Pediatr Surg 2014;24:9-13
Address for correspondence Augusto Zani, MD, PhD, MRCS, Department of Paediatric Surgery, University College London Institute of Child Health, London, WC1N 1EH, United Kingdom (e-mail: a.zani@ucl.ac.uk).

\section{Abstract \\ Keywords \\ - herniotomy \\ - herniorrhaphy \\ - minimal invasive surgery \\ - Europe}

Aim The aim of the study is to present views of pediatric surgeons on the optimal approach to inguinal hernias in children.

Methods A questionnaire was submitted to participants of EUPSA-BAPS 2012 and it was returned by 187 responders ( $85 \%$ senior surgeons) from 46 (27 European) countries.

Main Results Approximately $80 \%$ of the responders work in centers treating more than 100 cases/year. Approximately $79 \%$ of the responders routinely perform laparoscopy for other conditions, but of these, only $22 \%$ of the responders routinely perform laparoscopic inguinal hernia repair. Open approach is preferred by $83 \%$ of the responders. Of these, $79 \%$ of the responders favor unilateral approach, $12 \%$ of the responders contralateral, and $9 \%$ of the responders contralateral laparoscopic exploration via hernial sac. Considered received

May 15, 2013

accepted after revision

July 16, 2013

published online

September 2, 2013 (c) 2014 Georg Thieme Verlag KG

Stuttgart · New York
DOI http://dx.doi.org/ $10.1055 / \mathrm{s}-0033-1354586$. ISSN 0939-7248. 
advantages are the less risk of recurrence (66\%), less abdominal organ injury (31\%), less vas/ vessel injury (25\%), and speed (16\%). Laparoscopic approach is preferred by $17 \%$ of the respondents, of whom $58 \%$ perform laparoscopy at all ages, $15 \%$ only in younger than 1 year-old infants. Approximately $81 \%$ of the responders offer laparoscopy to both sexes, and $17 \%$ only in girls. Only $15 \%$ would do laparoscopy after incarceration. Purse string is the most popular (58\%), then laparoscopic-guided percutaneous closure (28\%), "flip-flap" (6\%), or other techniques (8\%). Proficiency is achieved after $50(14 \%), 25(48 \%), 10(32 \%)$, or 5 (6\%) laparoscopic hernia repairs. Considered advantages are less metachronous contralateral hernias (57\%), better cosmesis (41\%), easier technique (34\%), less vas/vessel injury (31\%), and less postoperative pain (22\%). A total of 50 responders are planning to start laparoscopic hernia repairs.

Conclusions Most pediatric surgeons favor open unilateral inguinal hernia repair. The role of laparoscopy in inguinal hernias is still controversial and yet to be accepted even amongst pediatric surgeons expert in laparoscopy.

\section{Introduction}

Although inguinal hernia is one of the most common conditions that pediatric surgeons encounter, ${ }^{1}$ there are still some controversies on different aspects of the management, for example, on the role of laparoscopy.

The aim of this study was to present current views of pediatric surgeons on the optimal approach to inguinal hernias in children.

\section{Methods}

A questionnaire was administered to delegates attending the EUPSA-BAPS Joint Congress (June 2012) and collected on the last day of the conference. Respondents were asked to fill in their position (Head of Department/Permanent Staff or Consultant/Trainee), hospital, and country of practice. The questionnaire focused on the surgical approach to pediatric inguinal hernias, including questions on minimally invasive approach (-Fig. 1).

The questionnaire was returned by 187 responders, from 46 countries including 19 countries from outside Europe (respondents, $n=34,18 \%$ ). Of the 165 respondents who declared their degree, there were $28(17 \%)$ heads of department, 113 (68\%) consultants, and 24 (15\%) trainees.

\section{Results}

A total of 149 (80\%) respondents work in centers treating more than 100 cases of inguinal hernia a year, whereas 32 (17\%) respondents treat 50 to 100 cases a year, and only 5 (3\%) respondents treat between 25 and 50 cases a year. Of the 149 respondents, 147 (79\%) routinely perform operative laparoscopy for other conditions, whereas $40(21 \%)$ respondents are not laparoscopists. The preferred approach for inguinal hernia repair is open for 155 (83\%) respondents, laparoscopic for 7 (4\%) respondents, and open or laparoscopic depending on age or gender in $25(13 \%)$ respondents. Interestingly, of the 147 laparoscopists, only 32 (22\%) repair inguinal hernias laparoscopically.

\section{Open Repair}

Of the 155 surgeons preferring the open approach, 145 (79\%) surgeons favor a unilateral approach when repairing a unilateral inguinal hernia, $22(12 \%)$ a bilateral approach, and $17(9 \%)$ a contralateral laparoscopic exploration via hernial sac. The considered advantages of open inguinal hernia repair compared with laparoscopic are reported in -Table 1.

\section{Laparoscopic Repair}

This approach is chosen by 32 (17\%) respondents, of whom only 7 (4\%) respondents favor it as the only approach and 25 (13\%) respondents opt for it depending on patient age and/or gender.

The majority of respondents $(n=30,58 \%)$ perform laparoscopic hernia repair at all ages, whereas $8(15 \%)$ respondents only in younger than 1-year-old infants, and $14(27 \%)$ in younger than 5 -year-old children. The majority of surgeons ( $n=37,81 \%)$, carry out laparoscopy on children of either sex, whereas $8(17 \%)$ surgeons perform laparoscopic repair only in girls and $1(2 \%)$ surgeon only in boys. Only nine (15\%) surgeons would do laparoscopy after inguinal hernia incarceration. Furthermore, eight surgeons perform laparoscopic repair in case of hernia recurrence and two if a hernia is found incidentally during surgery.

The preferred laparoscopic suturing technique is the purse string $(n=38,58 \%)$, followed by laparoscopic-guided percutaneous closure $(n=18,28 \%)$ and "flip-flap" $(n=4,6 \%)$. Moreover, 5 (8\%) respondents prefer other techniques, including intracorporeal Z-stitch, extracorporeal N-stitch, and needle technique.

Most surgeons ( $n=49,48 \%$ ) suggest that proficiency is achieved after 25 laparoscopic hernia repairs, whereas the others consider 50 ( $n=14,14 \%), 10(n=33,32 \%)$, or even just $5(n=6,6 \%)$ procedures the learning curve minimum.

The considered advantages of laparoscopic inguinal hernia repair compared with open approach are reported in -Table 2. 


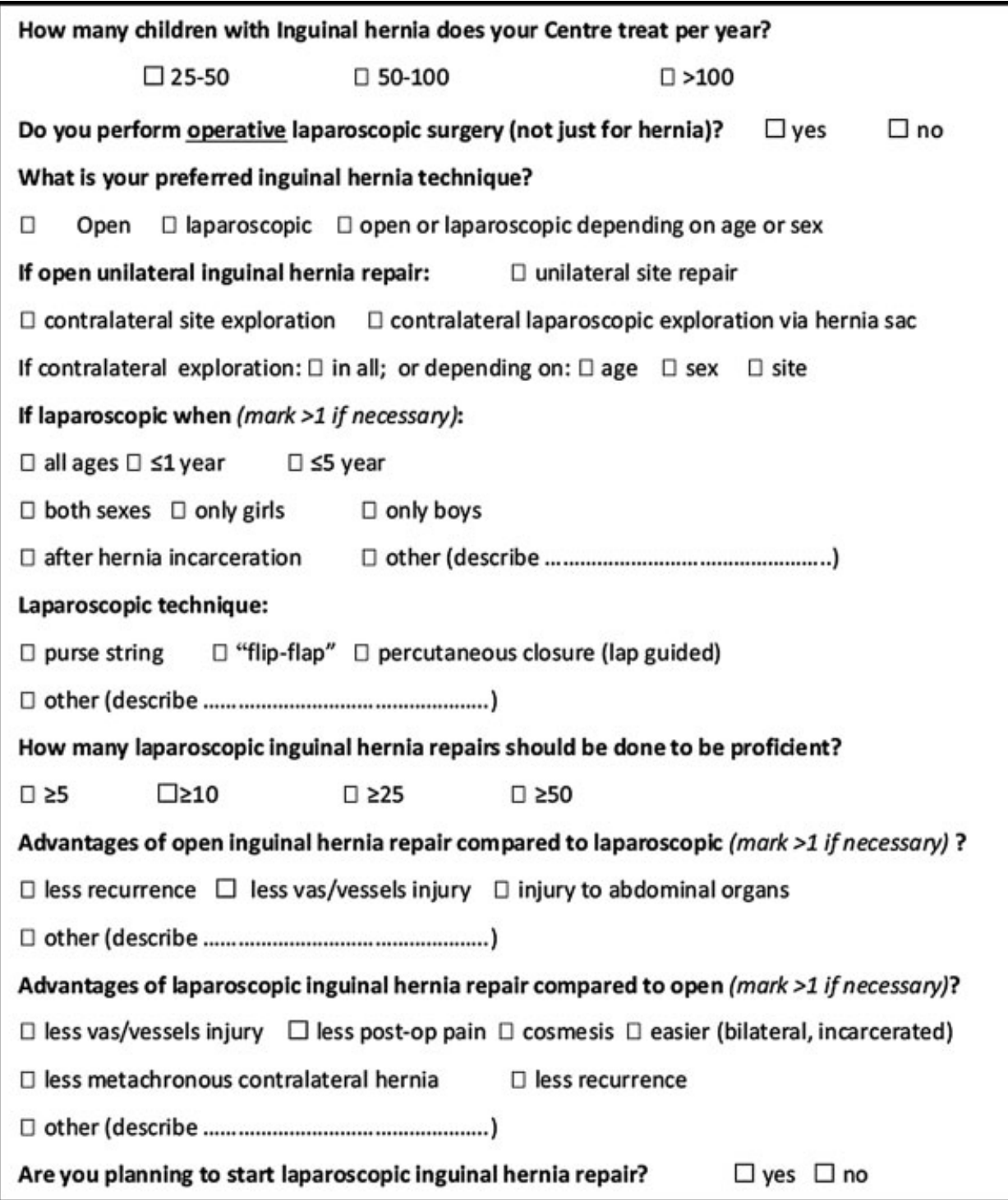

Fig. 1 The questionnaire administered to the delegates of the 2012 EUPSA-BAPS Joint Congress.

Finally, 50 responders are planning to start laparoscopic hernia repair shortly. Of these, 12 responders do not routinely perform operative laparoscopy for any surgical conditions.

\section{Discussion}

Our survey confirmed that in the last decades the number of infants or children with inguinal hernias operated in pediatric surgical centers has been increasing. This could be because of anesthetic preference and higher postoperative complications (e.g., recurrence) when the procedure is performed in adult general units. ${ }^{2-4}$

The results of this survey show that the percentage of respondents that routinely perform minimally invasive procedures in children is fairly high. However, it is interesting that only a small proportion of these pediatric laparoscopists would favor laparoscopy to repair inguinal hernias. The first case of laparoscopy in pediatric surgery was reported by Stephen Gans more than 40 years ago, when he used what he termed "peritoneoscopy" to verify the presence of a possible contralateral inguinal hernia introducing the scope via the hernial sac. ${ }^{5}$ Since then, it took a long time for smaller instruments and better video technologies to be available to be used in children, but from the 1990s onwards minimally invasive surgery in children has increasingly found its indications.

The vast majority of respondents favor an open inguinal approach that in case of unilateral hernia would be reserved to the affected side. Exploring the contralateral side has been routine practice for many surgeons, but many others have an opposite view on this, hence, this evergreen debate has not been solved, yet.

In 1953, Dr Robert E. Gross reported that he had abandoned the contralateral routine exploration in all subjects, as more frequently nothing had been found and he advised to open on the other side only if history or clinical examination were suggestive of a hernia. ${ }^{6}$ This approach has later been supported especially in boys by reports showing the risks of 
Table 1 Advantages of open inguinal hernia repair compared with laparoscopic ${ }^{a}$

\begin{tabular}{|l|l|l|}
\hline & $\begin{array}{l}\text { Number of } \\
\text { respondents }\end{array}$ & $\%$ \\
\hline Less risk of recurrence & 101 & 66 \\
\hline Less injury to abdominal organs & 48 & 31 \\
\hline Less risk for vas/vessels injury & 39 & 25 \\
\hline Quicker procedure & 25 & 16 \\
\hline Extraperitoneal approach & 6 & 4 \\
\hline Better cosmetic outcome & 6 & 4 \\
\hline Easier to perform & 5 & 3 \\
\hline Less invasive & 5 & 3 \\
\hline No need for lap equipment & 4 & 3 \\
\hline Less anesthesia & 4 & 3 \\
\hline $\begin{array}{l}\text { Teaching opportunity } \\
\text { for juniors }\end{array}$ & 3 & 2 \\
\hline Cheaper & 3 & 2 \\
\hline $\begin{array}{l}\text { No evidence for laparoscopic } \\
\text { repair }\end{array}$ & 2 & 1 \\
\hline Better for selected patients & 2 & \\
\hline
\end{tabular}

Note: Total number of respondents to this question $=153$.

${ }^{\mathrm{a}}$ More than one option allowed.

Table 2 Advantages of laparoscopic inguinal hernia repair compared with open ${ }^{\mathrm{a}}$

\begin{tabular}{|l|l|l|}
\hline & $\begin{array}{l}\text { Number of } \\
\text { respondents }\end{array}$ & $\%$ \\
\hline Less risk of metachronous hernia & 64 & 57 \\
\hline Better cosmetic outcome & 57 & 50 \\
\hline Easier to perform & 38 & 34 \\
\hline Less risk for vas/vessels injury & 35 & 31 \\
\hline Less postoperative pain & 25 & 22 \\
\hline Less risk of recurrence & 1 & 1 \\
\hline
\end{tabular}

Note: Total number of respondents to this question $=113$.

${ }^{a}$ More than one option allowed.

damaging the testis and surrounding structures when exploring an asymptomatic contralateral site. ${ }^{7-9}$ Chertin et al reported that a contralateral exploration seems unnecessary in girls too, because of their low risk of developing a contralateral inguinal hernia. ${ }^{10}$

A systematic review of the literature showed that the risk for a child to develop a contralateral hernia is approximately $7 \%$ and that 14 contralateral explorations would be required to prevent a metachronous hernia. ${ }^{1}$ On contrary, other authors, ${ }^{11,12}$ mainly laparoscopists, have advocated routine contralateral exploration justifying this approach by the high incidence of subsequent hernias if a contralateral patent processus vaginalis were encountered. Moreover, it has been demonstrated that laparoscopic contralateral groin explora- tion at the time of unilateral inguinal hernia repair is cost effective. ${ }^{13}$ Finally, it is interesting to compare the percentage of contralateral exploration extrapolated from our survey with that reported in two surveys conducted by the Section on Surgery of the American Academy of Pediatrics (AAP) in 1993 and 2003, respectively. ${ }^{14,15}$ According to the AAP surveys, most surgeons would explore the contralateral side in various individualized cutoff ages, beyond which they would no longer routinely explore, leaving the cohort of surgeons who would never do a contralateral exploration in boys very low (2\% in 1993 and $15 \%$ in 2003). Our survey is at variance from that conducted by the AAP but is more recent and reflects primarily the opinion of surgeons operating in Europe.

Laparoscopy for inguinal hernia repair is still not very popular in Europe, with only $17 \%$ of the respondents choosing this approach. Conversely, in the AAP surveys, the use of laparoscopy increased from $6 \%$ in 1993 to $37 \%$ in $2003 .{ }^{14,15}$

This reticence toward laparoscopy in Europe is even more striking given that this approach is favored by a small minority of surgeons who routinely use laparoscopy in their surgical practice. The main criticisms toward laparoscopic repair seem to be because of a perceived higher risk of hernia recurrence, higher risk to damage the surrounding structures and longer procedure time. However, according to a metaanalysis that comprises 3 randomized controlled trials and 4 observational clinical studies for a total 2,200 cases $(1,543$ laparoscopic vs. 657 open approach), laparoscopic hernia repair has similar operative time, length of hospital stay, recurrence, and complication rates compared with open herniotomy. ${ }^{16}$ Moreover, laparoscopic herniorrhaphy has a lower metachronous hernia rate when compared with open heniotomy. ${ }^{16}$ Furthermore, these findings have been confirmed by a later randomized controlled trial, where laparoscopic-assisted inguinal hernia repair performed using a Reverdin needle had similar hospital stay and recurrence rate but shorter operative time and lower testicular complication rate than the open approach. ${ }^{17}$

Only a minority of surgeons opt for laparoscopy in case of inguinal hernia incarceration, although Nah et al have described potential advantages of laparoscopic repair of incarcerated inguinal hernia, namely, fewer complications and avoiding the often challenging dissection of an edematous hernial sac, decreasing the risk of damaging the testicle and its vas and vessels. ${ }^{18}$

Different techniques have been proposed for laparoscopic hernia repair in children, the purse string being the most popular. Currently, there is no evidence in the literature that one is more advantageous than another.

The majority of respondents consider 10 to 25 the number of procedures needed to become competent to carry out a pediatric laparoscopic hernia repair. These figures are very close to that quoted by Bertozzi et al, who demonstrated a learning curve of a minimum of 20 procedures to achieve competency. ${ }^{19}$ These numbers could set the threshold for the 50 respondents who declared their intention to start performing laparoscopic inguinal hernia repair in the near future. In the modern era of surgery, to acquire the necessary 
skills for minimal invasive techniques, beginners are now encouraged to practice first on a simulator, then on patients. $^{20}$ This novel way of training could decrease the learning curve further and get the new generation of surgeons closer to minimal invasive surgery.

In conclusion, there are still some controversial aspects in the management of pediatric inguinal hernias. The majority of surgeons responding to our questionnaire would not explore a contralateral asymptomatic groin, even though there is still an open debate in the literature. Moreover, although laparoscopic surgery is becoming the standard of care for many pediatric conditions, at present, most pediatric surgeons in Europe favor an open unilateral repair for inguinal hernias. However, this picture might change as shown by a new interest recorded at the time of the survey and growing literature evidence on the subject.

\section{Conflict of Interest}

None.

\section{References}

1 Ron O, Eaton S, Pierro A. Systematic review of the risk of developing a metachronous contralateral inguinal hernia in children. Br J Surg 2007;94(7):804-811

2 Arul GS, Spicer RD. Where should paediatric surgery be performed? Arch Dis Child 1998;79(1):65-70, discussion 70-72

3 Borenstein SH, To T, Wajja A, Langer JC. Effect of subspecialty training and volume on outcome after pediatric inguinal hernia repair. J Pediatr Surg 2005;40(1):75-80

4 Surgical Advisory Panel. American Academy of Pediatrics. Guidelines for referral to pediatric surgical specialists. Pediatrics 2002;110(1 Pt 1):187-191

5 Gans SL, Berci G. Advances in endoscopy of infants and children. J Pediatr Surg 1971;6(2):199-233

6 Gross RE. Inguinal hernia. In: The Surgery of Infancy and Childhood. Philadelphia, PA: WB Saunders Co.; 1953:449-462
7 Surana R, Puri P. Is contralateral exploration necessary in infants with unilateral inguinal hernia? J Pediatr Surg 1993;28(8):10261027

8 Marulaiah M, Atkinson J, Kukkady A, Brown S, Samarakkody U. Is contralateral exploration necessary in preterm infants with unilateral inguinal hernia? J Pediatr Surg 2006;41(12):2004-2007

9 Steven M, Greene O, Nelson A, Brindley N. Contralateral inguinal exploration in premature neonates: is it necessary? Pediatr Surg Int 2010;26(7):703-706

10 Chertin B, De Caluwé D, Gajaharan M, Piaseczna-Piotrowska A, Puri P. Is contralateral exploration necessary in girls with unilateral inguinal hernia? J Pediatr Surg 2003;38(5):756-757

11 Holcomb GW III, Morgan WM III, Brock JW III. Laparoscopic evaluation for contralateral patent processus vaginalis: Part II. J Pediatr Surg 1996;31(8):1170-1173

12 Valusek PA, Spilde TL, Ostlie DJ, et al. Laparoscopic evaluation for contralateral patent processus vaginalis in children with unilateral inguinal hernia. J Laparoendosc Adv Surg Tech A 2006;16(6):650653

13 Lee SL, Sydorak RM, Lau ST. Laparoscopic contralateral groin exploration: is it cost effective? J Pediatr Surg 2010;45(4):793-795

14 Wiener ES, Touloukian RJ, Rodgers BM, et al. Hernia survey of the Section on Surgery of the American Academy of Pediatrics. J Pediatr Surg 1996;31(8):1166-1169

15 Antonoff MB, Kreykes NS, Saltzman DA, Acton RD. American Academy of Pediatrics Section on Surgery hernia survey revisited. J Pediatr Surg 2005;40(6):1009-1014

16 Yang C, Zhang H, Pu J, Mei H, Zheng L, Tong Q. Laparoscopic vs open herniorrhaphy in the management of pediatric inguinal hernia: a systemic review and meta-analysis. J Pediatr Surg 2011;46 (9):1824-1834

17 Shalaby R, Ibrahem R, Shahin M, et al. Laparoscopic Hernia Repair versus Open Herniotomy in Children: A Controlled Randomized Study. Minim Invasive Surg 2012;2012:484135

18 Nah SA, Giacomello L, Eaton S, et al. Surgical repair of incarcerated inguinal hernia in children: laparoscopic or open? Eur J Pediatr Surg 2011;21(1):8-11

19 Bertozzi M, Melissa B, Magrini E, Bini V, Appignani A. Laparoscopic herniorrhaphy in the pediatric age group: what about the learning curve? J Endourol 2013;27(7):840-844

20 Curry JI. 'See one, practise on a simulator, do one' - the mantra of the modern surgeon. S Afr J Surg 2011;49(1):4-6 